\title{
Digital Subtraction Angiographic Study of Circle of Willis in Indian Population in a Tertiary Super-Specialty Hospital
}

\author{
Muhammad Abdul Momen Khan 1 , Shakir Husain², Md. Shohidul Islam³, Md. Amir Hossain ${ }^{4}$ \\ ${ }^{1}$ Assistant Professor, Department of Interventional Neurology, National Institute of Neurosciences \& Hospital, Dhaka, Bangladesh; \\ ${ }^{2}$ Director of Neurointervention, Max super-specialty Hospital, New Delhi, India, Visiting Professor of Department of \\ Interventional Neurology, National Institute of Neurosciences \& Hospital, Dhaka, Bangladesh; ${ }^{3}$ Assistant Professor \\ of Neurology, Officer on Special Duty, Directorate general of Health Services, Ministry of Health \& Family \\ Welfare, Dhaka, Bangladesh; ${ }^{4}$ Assistant Professor, Department of Interventional Neurology, National \\ Institute of Neurosciences \& Hospital, Dhaka, Bangladesh
}

[Received: 21 January 2017; Revised: 6 March 2017; Accepted: 11 April 2017; Published: 1 July 2017]

\begin{abstract}
Background: Circle of Willis is an anastomotic polygon at the base of the brain which forms an important collateral network to maintain adequate cerebral perfusion. Changes in the normal morphology of the circle may causes the appearance and severity of symptoms of cerebrovascular disorders, such as aneurysms, infarctions and other vascular anomalies. Objectives: The aim of the present study was to analyses the anatomical variations of the circle of Willis by observing the variations in the cerebral arterial circle and was to clarify the clinical importance of these variations in certain forms of cerebrovascular diseases. Methodology: This cross-sectional study was conducted in the department of Neurointervention of Max Super-speciality Hospital, New Delhi, India and Neo multispeciality Hospital, Noida, Uttar Pradesh (UP), India during July 2016 to December 2016 for a period of six (6) months. Patients who were admitted in the Neurointervention department for digital subtraction angiography (DSA) were included in this study. The circle of Willis was then analyzed with the special reference to the complete or incomplete circle, any asymmetry in the configuration and variations in the size, and number of the component vessels, circle with multiple anomalies and absence, fenestration, duplication or triplication of any of the vessels. Results: Morphology and variations of the circle of Willis were studied in 74 patients undergone digital subtraction angiography (DSA). The normal pattern of circle of Willis was observed in $40(54.06 \%)$ cases and the remaining 34(45.94\%) cases had one or more variations; however, $24(70.58 \%)$ cases had variations in the anterior circulation and $10(29.42 \%)$ cases had variations in the posterior circulation. $17(50 \%)$ cases had variations on the right side compared to $13(38.24 \%)$ variations on the left side. $4(11.76 \%)$ cases had variations in the anterior communicating artery. Incomplete circle of Willis were found $12(35.28 \%)$ cases. Multiple variations were observed in 11 cases (32.35\%) in this study. In 4 cases anterior communicating artery aneurysms were observed. Conclusion: Variation of circle of Willis is common in this study of Indian population. [Journal of National Institute of Neurosciences Bangladesh, 2017;3(2): 99-105]
\end{abstract}

Keywords: Circle of Willis, variations, hypoplasia, aplasia

Correspondence: Dr. Muhammad Abdul Momen Khan, Assistant Professor, Department of Interventional Neurology, National Institute of Neurosciences \& Hospital, Dhaka, Bangladesh; Email: momen37cmc@gmail.com; Cell no.: +8801718738141

Conflict of interest: There is no conflict of interest relevant to this paper to disclose.

Funding agency: This research project was not funded by any group or any institution.

Contribution to authors: Khan MAM, Husain S, Islam MS, Hossain MA. was involved in study design, sample collection, patient's management, report analysis as well as manuscript writing.

How to cite this article: Khan MAM, Husain S, Islam MS, Hossain MA. Digital Subtraction Angiographic Study of Circle of Willis in Indian Population in a Tertiary Super-Specialty Hospital. J Natl Inst Neurosci Bangladesh, 2017;3(2): 99-105

Copyright: (C2017 Khan et al. Published by Journal of National Institute of Neurosciences Bangladesh. This article is published under the Creative Commons CC BY-NC License (https://creativecommons.org/licenses/by-nc/4.0/). This license permits use, distribution and reproduction in any medium, provided the original work is properly cited, and is not used for commercial purposes.

\section{Introduction}

Human brain and its complex vascular network may be viewed as a "three pound biological computer". It is composed of billions of specialized cells and circuits which make it the most vital organ in the human body. It is richly supplied with blood vessels which provide 
oxygen and other essential nutrients. Brain is supplied by two internal carotid and two vertebral arteries, connected by a central anastomosis- circulus arteriosus, which lie in the interpeduncular fossa at the base of brain ${ }^{1}$. Circle of Willis was named after a popular British Anatomist-Physician Thomas Willis who was the first to describe it completely ${ }^{2}$. The internal carotid artery divides into the anterior and middle cerebral arteries. The two anterior cerebral arteries are joined together by an anterior communicating artery. The basilar artery formed by the union of the two vertebral arteries, branches into two posterior cerebral arteries. The posterior communicating artery, a branch of internal carotid completes the arterial polygon by joining with the posterior cerebral artery. A classical arterial circle is bilaterally symmetrical and a complete ring of vessels ${ }^{1}$.

There is considerable individual variation in the pattern and caliber of vessels that make up the circulus arteriosus. Although a complete circular channel almost always exists, one vessel is usually sufficiently narrowed to reduce its role as a collateral route and the circle is rarely functionally complete. The haemodynamics of the circle are influenced by variations in the caliber of communicating arteries and in the segments of the anterior and posterior cerebral arteries which lie between their origins and their junctions with the corresponding communicating arteries. The greatest variation in caliber between individuals occurs in the posterior communicating artery, which is normally very small, so that only limited flow is possible between the anterior and posterior circulations ${ }^{3}$.

There may be variations in this typical configuration. Vessels generally vary in caliber; often they are hypoplastic, duplicated or even absent $t^{4}$. Several studies have shown that these variations play an important role in the development of cerebrovascular diseases 5 . Anatomical variations are probably genetically determined, develop in early embryonic stage and persist in post-natal life ${ }^{6}$. Any change in the normal morphology of the circle may condition the appearance and severity of symptoms of cerebrovascular disorders, such as aneurysms, infarcts and other vascular anomalies ${ }^{7}$. Arterial aneurysms such as berry aneurysms and arterial variations are interconnected. Aneurysms usually tend to occur at branches or at the bifurcations of cerebral arteries.

It is a well-established fact that the adequacy and compliance of collateral circulation plays a vital role in the course and severity of a cerebrovascular disorder.
Analysis and establishing the pattern of circle of Willis is one of the most important prerequisites of various diagnostic \& therapeutic neurovascular procedures. In case of complete unilateral vascular occlusion or thrombosis, expansion of anastomotic channels and collateral circulation in circle of Willis is life saving?.

A thorough knowledge of the variations of vessels is useful to neurologists and neurosurgeons in planning their shunt operations and the choice of the patients. These problems make it desirable to have an intimate knowledge of the variations in the anatomy of the cerebral arterial circle and try to correlate these anomalies to the occurrence of the certain common clinical conditions such as stroke, aneurysms and other arterio-venous malformations. The inferences obtained from this study are also useful to anatomists and radiologists in enhancing their knowledge in teaching and investigative procedures.

\section{Methodology}

This observational study was conducted in the department of Neurointervention of Max Super-speciality Hospital, New Delhi, India and Neo multispeciality Hospital, Noida, Uttar Pradesh (UP), India during July 2016 to December 2016 for a period of six (6) months. A total of 74 patients were admitted in the Neurointervention department for digital subtraction angiography (DSA) included in this study. Sampling technique was purposive. The angiogram machine was SIEMENS Artis Zee system and framing rate was $4 \mathrm{f} / \mathrm{sec}$ and for proper visualization of anterior communicating artery (AComA) caudal $15^{\circ}$ anterior-posterior $(\mathrm{A} / \mathrm{P})$ projection was encountered. Angiograms with gross pathology like arteriovenous malformations (AVM) were excluded from the study. The circle of Willis was then analyzed with the special reference to the following factors-whether the circle is complete or incomplete, any asymmetry in the configuration and variations in the size, and number of the component vessels, circle with multiple anomalies and absence, fenestration, duplication or triplication of any of the vessels. In present study, Kamath's observations have followed in defining hypoplasia and fixed arterial diameters of less than $1 \mathrm{~mm}$ and $0.5 \mathrm{~mm}$ as hypoplastic for cerebral and communicating arteries respectively and data drawn in data collection sheet. All collected data were checked, edited and analyzed by using computer based SPSS software version 16.0. Data were presented by frequency distribution and percentage. 


\section{Results}

Morphology and variations of the circle of Willis were studied in 74 patients undergone digital subtraction angiography (DSA). Age and gender distribution among the subjects studied, out of 74 cases, $51(68.92 \%)$ male cases and $23(31.08 \%)$ female cases were observed, most of the patients $20(27.02 \%)$ belonged to 50-59 years age group, among them about $15(20.27 \%)$ of the male and $5(6.76 \%)$ of the female. Lowest number of patient $(5.40 \%)$ belonged to 20 to 29 years age group (Table 1$)$.

Table 1: Distribution of the Study Population by Age and Gender ( $\mathrm{N}=74)$

\begin{tabular}{lccc}
\hline Age Group & \multicolumn{2}{c}{ Gender } & Total \\
\cline { 2 - 3 } & Male & Female & \\
\hline 10 to 9 Years & $5(6.76 \%)$ & $2(2.70 \%)$ & $7(9.46 \%)$ \\
20 to 29 Years & $3(4.05 \%)$ & $1(1.35 \%)$ & $4(5.40 \%)$ \\
30 to 39 Years & $6(8.10 \%)$ & $2(2.70 \%)$ & $8(10.80 \%)$ \\
40 to 49 Years & $10(13.51 \%)$ & $7(9.46 \%)$ & $17(22.97 \%)$ \\
50 to 59 Years & $15(20.27 \%)$ & $5(6.76 \%)$ & $20(27.02 \%)$ \\
60 to 69 Years & $7(9.46 \%)$ & $4(5.40 \%)$ & $11(14.87 \%)$ \\
70 to 79 Years & $5(6.76 \%)$ & $2(2.70 \%)$ & $7(9.46 \%)$ \\
Total & $\mathbf{5 1 ( 6 8 . 9 2 \% )}$ & $\mathbf{2 3 ( 3 1 . 1 \% )}$ & $\mathbf{7 4 ( 1 0 0 . 0 \% )}$ \\
\hline
\end{tabular}

It was observed out of 74 cases, 51(68.92\%) cases were male, among them $28(37.83 \%)$ cases had normal circle of Willis and 23(31.07\%) cases had abnormal circle of Willis. Among 23(31.06) cases female, 12(16.21\%) cases had normal circle of Willis and $11(14.85 \%)$ cases had abnormal circle of Willis. Male to female ratio was 2.21:1(Table 2).

Table 2: Distribution of the Study Population by Variation of Circle of Willis $(n=74)$

\begin{tabular}{lccc}
\hline Gender & \multicolumn{2}{c}{ Gender } & Total \\
\cline { 2 - 3 } & Normal & Abnormal & \\
\hline Male & $28(37.83 \%)$ & $23(31.07 \%)$ & $51(68.92 \%)$ \\
Female & $12(16.21 \%)$ & $11(14.85 \%)$ & $23(31.08 \%)$ \\
Total & $\mathbf{4 0 ( 5 4 . 0 6 \% )}$ & $\mathbf{3 4 ( 4 5 . 9 4 \% )}$ & $\mathbf{7 4 ( 1 0 0 . 0 \% )}$ \\
\hline
\end{tabular}

Out of 34 variations, 24(70.58\%) variations were observed in the anterior circulation and 10(29.42\%) variations were observed in the posterior circulation. More variations were observed on the right side than on the left side. 17(50\%) variations were observed on the right side as compared to $13(38.24 \%)$ variations on the left side. $4(11.76 \%)$ variations were observed in the anterior communicating artery (Table 3 ).
Table 3: Distribution of the Study Population by Variation $(\mathrm{n}=34)$

\begin{tabular}{lcccc}
\hline Group & Name of Artery & Distribution & Frequency & Percentage \\
\hline $\begin{array}{l}\text { Anterior } \\
\text { Circulation }\end{array}$ & ACA(A1) & Right- 4 & 7 & 20.58 \\
& & Left- 3 & & \\
& AComA & & 4 & 11.76 \\
& PComA & Right- 7 & 13 & 38.23 \\
& & Left- 6 & & \\
Posterior & PCA(P1) & Right- 6 & 10 & 29.41 \\
$\begin{array}{l}\text { Circulation } \\
\text { Total }\end{array}$ & & Left- 4 & & \\
& & Right-17 & $\mathbf{3 4}$ & $\mathbf{1 0 0 . 0}$ \\
& & Left- 13 & & \\
\hline
\end{tabular}

$\mathrm{ACA}(\mathrm{A} 1)=$ Anterior cerebral artery; $\mathrm{AComA}=\mathrm{Anterior}$ communicating Artery; PComA=Posterior communicating artery; $\mathrm{PCA}=$ Posterior Communicating Artery

Anterior cerebral artery variations were observed in $7(20.58 \%)$ cases among the 34 variations. Hypoplastic (string like) A1 segment in 4(11.76\%) cases. In 1 case it was on the left side and in 3 cases on the right side. Aplastic A1 segment in 3(8.82\%) cases, among them 1 case on the right side and 2 cases on the left side. Variation in anterior communicating artery were observed in $4(11.76 \%)$ cases. Complete absence of anterior communicating artery were observed in $2(5.88 \%)$ cases, duplication were observed in $2(5.88 \%)$ cases. Highest number of variations was observed in posterior communicating artery; $13(38.23 \%)$ variations among the 34 variations were found to be exclusively in posterior communicating artery. Complete absence and hypoplasia (string like) were the variations observed in this artery. 3(8.82\%) cases had complete absence of posterior communicating artery. In 1 case it was observed on the right side and in 2 cases on the left side. $10(29.41 \%)$ cases had a hypoplastic (string like) posterior communicating artery. In 6 cases it were on the right side and 4 cases it was observed on the left side. Among the 34 variations, 10(29.42\%) variations were observed in posterior cerebral arteries. Absence of P1 segment was observed in 4(11.76) cases among them $2(5.88 \%)$ cases on the right side and $2(5.88 \%)$ cases on the left side. Hypoplastic P1segment were observed in 6(17.64\%) cases among them 4(11.76\%) cases on the right side and $2(5.88 \%)$ cases on the left side (Table 4).

Among 34 variations, 12 circles (35.28\%) were incomplete and majority cases $(23.52 \%)$ were in anterior circulation and rest of cases $(11.76 \%)$ were in posterior circulation (Table 5). 
Table 4: Distribution of the study population by name of artery, nature, distribution, number and percentage of variation with variants $(n=34)$

\begin{tabular}{lccc}
\hline Name of ArterNature and distribution & Frequency Percentage \\
\hline ACA(A1) & Hypoplastic(Left)-1 & & \\
& Hypoplastic(Right)-3 & 4 & 11.76 \\
ACA(A1) & Aplastic(Left)-2 & & \\
& Aplastic(Right)-1 & 3 & 8.82 \\
AComA & Duplication & & \\
& $\quad$ Aplastic & 2 & 5.88 \\
PComA & Hypoplastic(Left)-4 & 2 & 5.88 \\
& Hypoplastic(Right)-6 & 10 & 29.41 \\
PComA & Aplastic(Left)-2 & & \\
& Aplastic(Right)-1 & 3 & 8.82 \\
PCA(P1) & Hypoplastic(Left)-2 & & \\
& Hypoplastic(Right)-4 & 6 & 17.64 \\
PCA(P1) & Aplastic(Left)-2 & & \\
& Aplastic(Right)-2 & 4 & 11.76 \\
Total & & $\mathbf{3 4}$ & $\mathbf{1 0 0 . 0}$ \\
\hline
\end{tabular}

$\mathrm{ACA}(\mathrm{A} 1)=$ Anterior cerebral artery; $\mathrm{ACom} \mathrm{A}=\mathrm{Anterior}$ communicating Artery; PComA=Posterior communicating artery

Table 5: Distribution of the study population by the pattern of incomplete circle of Willis $(n=34)$

\begin{tabular}{lccc}
\hline Name of Artery & distribution & \multicolumn{2}{c}{ Frequency } \\
\hline ACA(A1) & Left-2 & 3 & 8.82 \\
& Right-1 & & \\
AComA & & 2 & 5.88 \\
PComA & Left-2 & 3 & 8.82 \\
& Right-1 & & \\
PCA(P1) & Left-2 & 4 & 11.76 \\
Total & Right-2 & & \\
\hline
\end{tabular}

Out of total 34 variations in this series, multiple anomalies were observed in $11(32.35 \%)$ circles. Among the multiple anomalies $7(20.58 \%)$ circles had two anomalies. More than two anomalies were observed in $4(11.76 \%$ ) circles (Table 6$)$..

\section{Discussion}

The circle of Willis and its branches are subjected to numerous variations. The variations not only differ from person to person but also on the two sides of the same individual. The prevalence of the 'typical or classic circle', the "normal" textbook polygon ranges from $4.6 \%$ to $72.2 \%{ }^{10} \mathrm{~A}$ possible reason for the wide range may be due to the diversity in nomenclature and the criteria used to define hypoplastic vessels. In present study Kamath's observations have followed in defining hypoplasia and fixed arterial diameters of less than $1 \mathrm{~mm}$ and $0.5 \mathrm{~mm}$ as hypoplastic for cerebral and communicating arteries respectively ${ }^{11}$. Inspite of numerous studies conducted over several decades in several countries employing various methods across different geographical and racial background, there is no clarity as to how and why the variations occur. The wide range in the prevalence of the typical configuration among various races warrants further researches to confirm the influence of genetic, regional, environmental and hemodynamic factors or the combination of these factors. Many theories had been put forward to explain the variations of the circle of Willis. Lazorthes et $\mathrm{al}^{12}$ stated that the variations in the caliber of the segments were due to amplitude of neck movements in the later years of life. The genetic factors are very important, although random biochemical or physical factors could also be responsible ${ }^{13}$. Milenkovic et $\mathrm{al}^{14}$ also observed that anomalies and variations of the circle of Willis are

Table 6: Distribution of Study Population by Circle of Willis with Multiple Anomalies ( $\mathrm{n}=34)$

\begin{tabular}{|c|c|c|c|c|c|}
\hline Pattern of & Name of Artery & Number & Total number & $\begin{array}{l}\text { Percentage of } \\
\text { variants }(n=34)\end{array}$ & $\begin{array}{c}\text { Total } \\
(\%)\end{array}$ \\
\hline \multirow{4}{*}{$\begin{array}{l}\text { Anomalies } \\
\text { Two(2) Anomalies }\end{array}$} & Right A1(Aplastic) with & 1 & & 2.94 & \\
\hline & dominant AComA & & & & \\
\hline & $\begin{array}{l}\text { Right P1(Hypoplastic) with } \\
\text { dominant PComA }\end{array}$ & 4 & 7 & 11.76 & 20.58 \\
\hline & $\begin{array}{l}\text { Right P1(Aplastic) with } \\
\text { dominant PComA }\end{array}$ & 2 & & 5.88 & \\
\hline \multirow{3}{*}{$\begin{array}{l}\text { More than two(2) } \\
\text { Anomalies }\end{array}$} & Left P1 (Hypoplastic) with & 2 & & 5.88 & \\
\hline & dominant PComA with duplicated AComA & & & & \\
\hline & $\begin{array}{l}\text { Left P1(Aplastic)with } \\
\text { dominant PComA with Right A1 (Hypoplastic) }\end{array}$ & 2 & 4 & 5.88 & 11.76 \\
\hline Total & & & 11 & & 32.35 \\
\hline
\end{tabular}


probably genetically determined and they develop in the early embryonic stage and persists into post-natal life. Van-Overbeeke et $\mathrm{al}^{15}$ observed that the variations in posterior segments of the circle were due to developmental modifications with increased functional demands in connection with rapid growth of occipital lobes. Cerebrovascular diseases such as stroke, aneurysms, internal carotid artery occlusion, unilateral flow restrictive external carotid artery disease together with their signs and symptoms grossly depend upon the variations of the anatomical pattern of circle of Willis.

Rogers observed that the circle of Willis is not an equalizer and distributor of blood from different sources, there being no mingling of blood from different sources in the circle under normal circumstances. It's functions as an anastomosis and offers a potential shunt under abnormal conditions such as might occur during an occlusion or spasm $^{16}$. The state of the circle becomes important in determining the adequacy of the brain circulation. The possibility of bypass/shunts in occlusion of one of the cerebral vessels and the adequacy of recovery or lack of recovery after vascular occlusions may be explained in part by variations in the anatomy of the circle of Willis ${ }^{17}$

Age and gender distribution among the subjects studied, most of the patients were male $51(68.92 \%)$ and majority of the patients $20(27.02 \%)$ belonged to $50-59$ years age group, among them about $15(20.27 \%)$ of the male and $5(6.76 \%)$ of the female. Lowest patient $4(5.40 \%)$ belonged to 20-29 years age group.

In the present study, out of 74 cases, 40 cases $(54.06 \%)$ had normal and 34 cases $(45.94 \%)$ had abnormal circle of Willis. It was observed 51 cases $(68.92 \%)$ were male, among them 28 cases $(37.83 \%)$ had normal circle of Willis and 23 cases $(31.07 \%)$ had abnormal circle of Willis. Among 23(31.06\%) female cases, 12 cases $(16.21 \%)$ had normal circle of Willis and 11 cases $(14.85 \%)$ had abnormal circle of Willis. Thus the findings of the present study are approximately similar to the findings of cadaveric study of Alpers et $\mathrm{a}^{18}$, Raja Reddy et $\mathrm{al}^{9}$, Stephen and $\mathrm{John}^{19}$, Raghavendra et $\mathrm{al}^{20}$ with respect to normal pattern of circle.

Out of 34 variations, 24(70.58\%) variations were observed in the anterior circulation and 10 variations $(29.42 \%)$ in the posterior circulation. More variations were observed on the right side than on the left side. $17(50 \%)$ variations were observed on the right side as compared to $13(38.24 \%)$ variations on the left side. 4 variations $(11.76 \%)$ were observed in the anterior communicating artery. One cadaveric study done by
Raghvendra et $\mathrm{al}^{20}$ observed that variations were approximately twice as common in the posterior circulation (15 cases, 68.2\%) as compared to the anterior circulation ( 7 cases, $31.8 \%$ ) which differs with present study with respect of location and variation.

Anterior cerebral artery variations were observed in 7 cases $(20.58 \%)$ among the 34 variations. Hypoplastic (string like) A1 segment in 4 cases (11.76\%). In 1 case it was on the left side and in 3 cases on the right side. Aplastic A1 segment in 3 cases (8.82\%), among them 1 case on the right side and 2 cases on the left side. Thus, the findings of the present study are approximately similar to the cadaveric study findings of Lippert ${ }^{22}(10 \%)$, Arthur et a ${ }^{23}(10.4 \%)$, Morris ${ }^{25}$ $(12 \%)$, Raghavendra et $\mathrm{a}^{20}(13.65 \%)$ with respect to hypoplastic A1 segment of anterior cerebral artery.

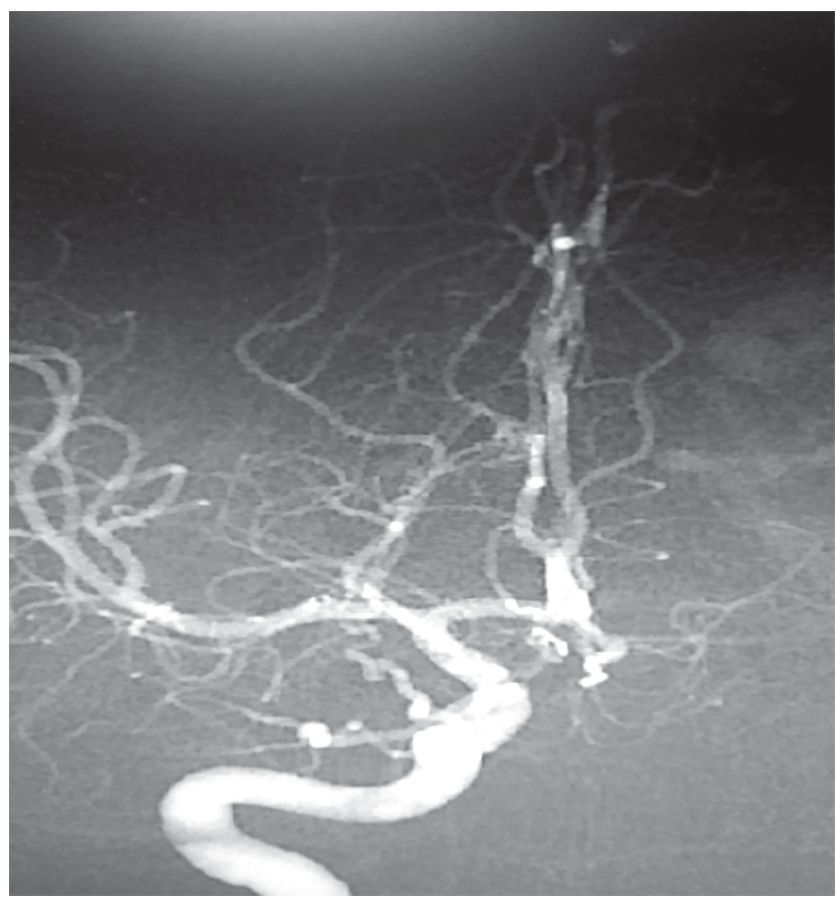

Figure I: Hypoplastic Left A1

Variation in anterior communicating artery were observed in $4(11.76 \%)$ cases. Complete absence of anterior communicating artery was observed in $2(5.88 \%)$ cases. A higher incidence $(9.1 \%)$ of absence of anterior communicating artery had been observed in Raghavendra et $\mathrm{al}^{20}$ cadaveric study, this is probably due to common carotid artery compression test was not done during DSA which signifies functional patency of communicating arteries though anatomical existence, duplication were observed in 2 cases (5.88\%). Thus, the findings of the present study are approximately similar to the cadaveric study findings reported by Jain and $\mathrm{Kumar}^{11}$ in $5.5 \%$ cases and Raghavendra et $\mathrm{al}^{20}$ in $4.5 \%$ cases with respect to duplication of anterior 
communicating artery.

Highest number of variations was observed in posterior communicating artery. $13(38.23 \%)$ variations among the 34 variations were found to be exclusively in posterior communicating artery. Complete absence and hypoplasia (string like) were the variations observed in this artery. 3(8.82\%) cases had complete absence of posterior communicating artery. In 1 case it was observed on the right side and in 2 cases on the left side. This findings vary with cadaveric study of Raghavendra et $\mathrm{al}^{20}(18.2 \%) .10$ cases $(29.41 \%)$ had a hypoplastic (string like) posterior communicating artery. In 6 cases it were on the right side and 4 cases it was observed on the left side (Table-IV). The findings of the present study are approximately similar to the cadaveric study findings of Pedroza et al. $(34 \%)^{24}$ and Raghavendra et al $(31.8 \%)^{20}$.

Among the 34 variations, 10(29.42\%) variations were observed in posterior cerebral arteries. Absence of P1 segment were observed in 4(11.76) cases among them $2(5.88 \%)$ cases on the right side and 2 cases $(5.88 \%)$ on the left side. Thus, the findings of present study are approximately similar to the cadaveric study findings reported by Raghavendra et $\mathrm{a}^{20}(4.55 \%)$. Hypoplastic P1segment was observed in 6 cases (17.64\%) among them 4 cases $(11.76 \%)$ on the right side and 2 cases $(5.88 \%)$ on the left side. Thus, the findings of present study are approximately similar to the cadaveric study findings reported by P N Jain and $\operatorname{Kumar}^{21}(16 \%)$ and Morris $^{25}(18.0 \%)$.

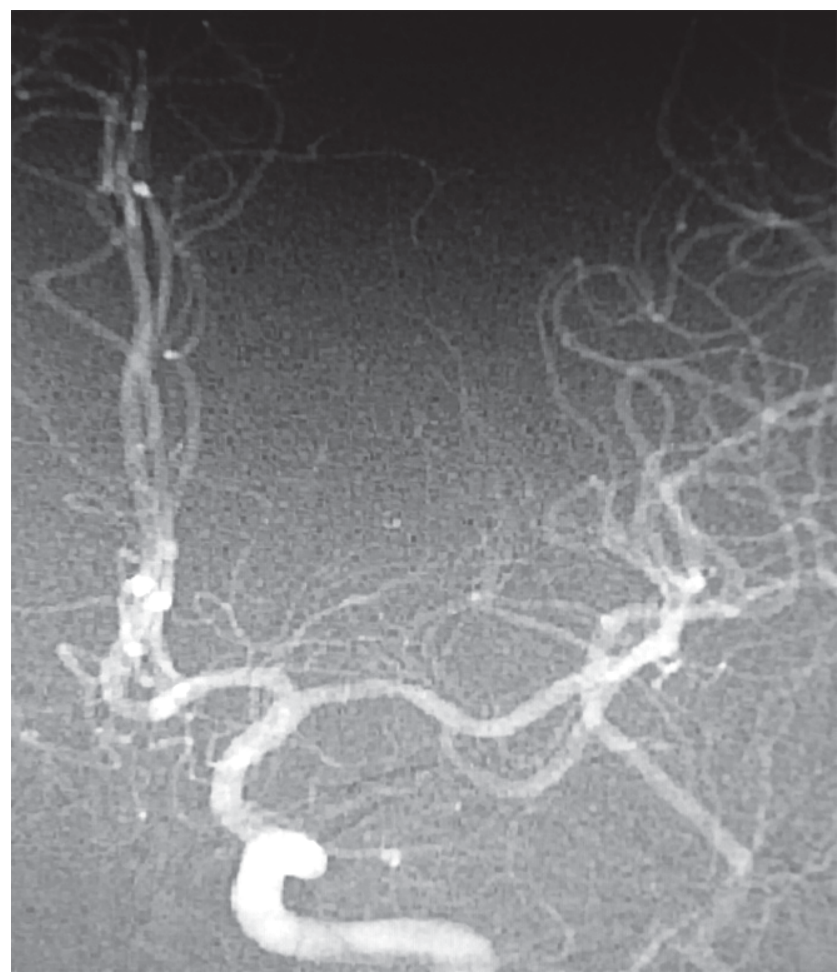

Figure II: Incomplete circle of Willis with dominant AComA
Among 34 variations, 12 circles $(35.28 \%)$ were incomplete and majority cases $(23.52 \%)$ were in anterior circulation and rest of cases $(11.76 \%)$ were in posterior circulation. Thus, the findings of present study differs with the cadaveric study findings reported by Lazorthes et al ${ }^{12}(17 \%)$ and Iqbal1 $(6.0 \%)$. Out of total 34 variations in this series, multiple anomalies were observed in 11(32.35\%) circles. Among the multiple anomalies 7(20.58\%) circles had two anomalies. More than two anomalies were observed in $4(11.76 \%)$ circles. Thus, the findings of present study are approximately similar to the cadaveric study findings reported by Iqbal ${ }^{1}(28 \%)$.

Limited number of cases and limited center based study, failure to do carotid compression test in all cases and contrast resolution of single panel angiography are the main limitations of present study.

\section{Conclusion}

In the present study the prevalence of variations is approximately twice in the anterior circulation compared to posterior circulation. The variations are more prevalent on the right side than on the left side. Incomplete circle of Willis is also found. Multiple variations is observed in this study. Anterior communicating artery aneurysms is observed. The most variable artery in the present study is posterior communicating artery, where the most common variation is hypoplastic posterior communicating artery. A thorough knowledge of the vascular variants will increase the success of the procedure. The anomalies of the circle of Willis play an important role in the occurrence, manifestation of symptoms, treatment options and recovery process of certain cerebrovascular disorders viz., stroke, and aneurysms. The knowledge of these anomalies will help the clinicians to understand pathophysiology, angio-architecture and haemodynamics of the lesions.

\section{References}

1. Iqbal S. A Comprehensive Study of the Anatomical Variations of the Circle of Willis in Adult Human Brains. J Clin Diagn Res. 2013;7(11):2423-27

2. Samuel M. Wolpert. History of the circle of Willis. Am J Neuroradiol. 1997; 18;1033-34

3. Susan Standring, editor in chief. Gray's Anatomy: The Anatomical Basis of Clinical Practice. 40th ed. London, UK: Churchill Livingstone Elsevier; 2008: 248-52

4. Alpers BJ, Berry RG,Paddison RM. Anatomical studies of the circle of Willis in normal brain. Arch Neurol Psychiat.1959; 81: $409-18$

5. Hoksbergen AW, Fulesdi B, Legemate AD, Csiba I. Collateral configuration of the circle of Willis: Transcranial color-coded duplex ultrasonography and comparison with postmortem anatomy. Stroke. 2003; 31: 1346-51 
6. Crompton MR. The pathology of ruptured middle cerebral aneurysms with special reference to the differences between the sexes. Lancet. 1962;2:421-25

7. Riggs HE, Rupp C. Variation in form of circle of Willis. The relation of the variations to collateral circulation: anatomic analysis. Arch Neurol 1963; 8: 24-30

8. Stehbens WE. Etiology of intracranial berry aneurysms. J Neurosurg. 1989; 70(6): 823-31

9. Reddy DR, Prabhakar V, Rao BD. Anatomical study of circle of Willis. Neurology India. 1972;20(1):8

10. Windle BCA. On the arteries forming circle of willis. J. Anat and Phy. 1888; 22:289

11. Kamath S. Observations on the length and diameter of the vessels forming the circle of Willis. J Anat.1981; 133: 419-23

12. Lazorthes G, Gouaze A, Santini JJ, Salamon G. The arterial circle of the brain (circulus arteriosus cerebri). Anatomica Clinica. $1979 ; 1: 241-57$

13. Fields WA. Aortao cranial occlusive vascular disease (stroke). Ciba Found Symp. 1974; 26 (4):3-31

14. Milenkovic Z, Vucetic R, Puzic M. Asymmetry and anomalies of the circle of Willis in fetal brain. Microsurgical study and functional remarks. Surg Neurol. 1985; 24(5): 563-70

15. Van Overbeeke JJ, Hillen B, Tulleken CA. A comparative study of the circle of Willis in fetal and adult life. The configuration of posterior bifurcation of the posterior communicating artery. J Anat. 1991; 176:45-54

16. Rogers L. The function of the circulus arteriosus of Willis.
Brain. 1946; 70: 171-78

17. De Silva KRD, Silva R, Gunasekera WSL, Jeyesekera RW. Prevalence of typical circle of Willis and the variation in the anterior communicating artery: A study of a Sri Lankan population. Ann Indian Acad Neurol. 2009; 12(3): 157- 61

18. Alpers BJ, Berry RG, Paddison RM. Anatomical studies of the circle of Willis in normal brain. Arch Neurol. 1959; 8(1): 409-18 19. Stephen PL, John Cerebral Angiography: The anterior cerebral disease. Cited by Henry J M Barnett, Mohr JP and Bennett M Stein. Stroke. Pathophysiology, Diagnosis and Management. 2nd ed. New York: Churchill Livingstone. 1991; 217-19

20. Raghavendra, Shirol VS, Daksha Dixit, Anil Kumar Reddy Y, Desai SP. Circle of Willis and its variations; morphometric study in adult Human cadavers. Int $J$ Med Res Health Sci. 2014;3(2):394-400

21. Jain PN, Kumar V, Thomas RJ, Longia GS. Anomalies of human cerebral arterial circleof Willis. J Anat 1990; 39(2): 137.

22. Lippert H, Padst R. Arterial variations in man. Munich, Germany: J F Bergmann Verlag 1985: 92 - 93

23. Arthur G. Kane, William P. Dillon. Reduced Caliber of the Internal Carotid Artery: A Normal Finding with Ipsilateral Absence or Hypoplasia of the A1 Segment. Am J Neuroradiol 1996; 7(3): 1295-1300

24. Alfred Pedroza, Manuel Dujovny, Jose Cabezudo Artero. Microanatomy of the posterior communicating artery. J Neurosurg 1987; 21 (2): 228-35

25. Morris P. Practical Neuroangiography. 3rd Ed. Philadelphia: Lippincott Williams \& Wilkins, 2013:114-31 